Erratum

Anaesthesist $2021 \cdot 70$ (Suppl 1):S76

https://doi.org/10.1007/s00101-021-00951-6

Online publiziert: 13. April 2021

(c) Springer Medizin Verlag $\mathrm{GmbH}$, ein Teil von Springer Nature 2021

Thomas Schmoch ${ }^{1,2}$ für SepNet Study Group - Thorsten Brenner ${ }^{2}$ für SepNet Study Group - Andrea Becker-Pennrich ${ }^{3}$ für SepNet Study Group ·

Ludwig Christian Hinske ${ }^{3,4}$ für SepNet Study Group · Markus A. Weigand' für SepNet Study Group · Josef Briegel ${ }^{3}$ für SepNet Study Group · Patrick Möhnle ${ }^{3}$ für SepNet Study Group

' Klinik für Anästhesiologie, Universitätsklinikum Heidelberg, Heidelberg, Deutschland

${ }^{2}$ Klinik für Anästhesiologie und Intensivmedizin, Universitätsklinikum Essen, Essen, Deutschland

${ }^{3}$ Klinik für Anästhesiologie und Abteilung für Transfusionsmedizin, Zelltherapeutika und

Hämostaseologie, LMU Klinikum München, München, Deutschland

${ }^{4}$ Institut für medizinische Informationsverarbeitung, Biometrie und Epidemiologie, LMU München,

München, Deutschland

\title{
Erratum zu: Therapie der sepsisinduzierten Koagulopathie. Ergebnisse einer deutschlandweiten Umfrage auf Intensivstationen
}

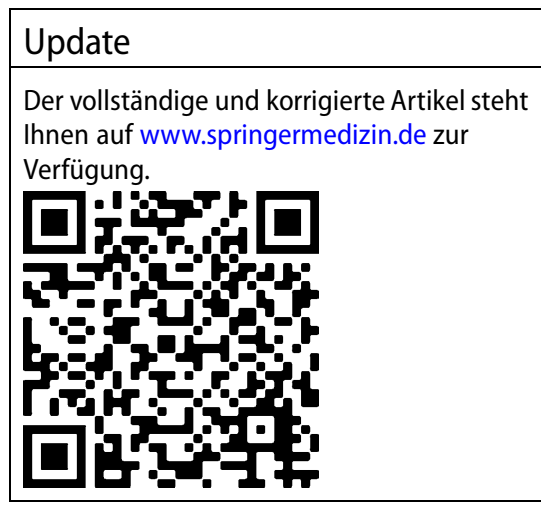

Erratum zu:

Anaesthesist 2021

https://doi.org/10.1007/s00101-021

00916-9

In dem ursprünglichen Artikel wurde an einigen Stellen eine falsche Abkürzung für „partielle Thromboplastinzeit“ verwendet. Korrekt wird die „partielle Thromboplastinzeit“ als PTT abgekürzt.

Dies wurde nun korrigiert.

\section{Korrespondenzadresse}

Dr. med. Thomas Schmoch

Klinik für Anästhesiologie und Intensivmedizin, Universitätsklinikum Essen

Hufelandstraße 55, 45147 Essen, Deutschland thomas.schmoch@uk-essen.de 